\title{
Performance of a novel dialyzer containing a fluorinated polyurethane surface-modifying macromolecule in patients with end-stage kidney disease
}

\section{Jill M Meyer}

Balboa Nephrology Medical Group

Dylan Steer

Balboa Nephrology Medical Group

Lisa A Weber

Via Christi Hospital on Saint Francis

Abeer A Zeitone

Fresenius Medical Care North America

Mayuri Thakuria ( $\square$ mayuri.thakuria@fmc-na.com )

Fresenius Medical Care North America https://orcid.org/0000-0003-4513-6289

Chiang-Hong Ho

Fresenius Medical Care North America

\section{Shakil Aslam}

Fresenius Medical Care North America

\section{Claudy Mullon}

Fresenius Medical Care North America

Robert J Kossmann

Fresenius Medical Care North America

\section{Research article}

Keywords: Dialyzer, Chronic hemodialysis, $\beta 2$-microglobulin, Surface-modifying macromolecule

Posted Date: May 12th, 2020

DOl: https://doi.org/10.21203/rs.3.rs-25165/v1

License: (c) (1) This work is licensed under a Creative Commons Attribution 4.0 International License. Read Full License 


\section{Abstract}

Background By inhibiting the adsorption of protein and platelets, surface-modifying macromolecules (SMMs) may improve the hemocompatibility of hemodialyzers. This trial aims to assess the performance and safety of a novel dialyzer with a fluorinated polyurethane SMM, Endexo ${ }^{\mathrm{TM}}$, blended into the membrane during manufacturing.

Methods This prospective, sequential, multicenter, open-label study enrolled adults prescribed thriceweekly hemodialysis. After completing 12 hemodialysis sessions with an Optiflux ${ }^{\circledR}$ F160NR dialyzer, patients received 38 sessions with the dialyzer with Endexo. Evaluated parameters included the extent of removal of urea, albumin, and $\beta 2$-microglobulin ( $\beta 2 \mathrm{M})$, as well as complement activation.

Results Twenty-three patients received 268 hemodialysis treatments during the Optiflux period, and 18 patients continued on to receive 664 hemodialysis treatments during the Endexo period. Mean treatment times (208 vs $205 \mathrm{~min}$ ), blood flow rates ( 447.7 vs $447.5 \mathrm{~mL} / \mathrm{min}$ ), dialysate flow rates (698.5 vs 698.0 $\mathrm{mL} / \mathrm{min})$, urea reduction ratio ( $82 \%$ vs $81 \%$ ) and spKt/V (2.1 vs 1.9$)$ were comparable for the Endexo and Optiflux periods, respectively. No overt complement activation was observed. Both dialyzers were associated with comparable mean increases in serum albumin levels from pre- to post- hemodialysis (Optiflux: 7.9\%; Endexo: 8.0\%). The corrected mean $\beta 2 \mathrm{M}$ removal rate was $47 \%$ higher during the Endexo period $(67.73 \%)$. Three serious adverse events were reported, none of them device-related.

Conclusions The performance of the novel dialyzer with Endexo was generally comparable to the Optiflux dialyzer, while exhibiting a higher $\beta 2 \mathrm{M}$ removal rate. Additional studies are needed to determine whether this novel dialyzer can be incorporated into heparin-free hemodialysis approaches.

\section{Introduction}

Globally, it is estimated that more that 3.7 million individuals will receive renal replacement therapy for end-stage kidney disease (ESKD) in 2020 [1], and that the vast majority of those patients will receive maintenance hemodialysis. In the absence of an ideal dialyzer-one capable of clearing all uremic toxins while allowing retention of beneficial plasma components and molecules-the selection of a dialyzer should be tailored to the needs of the patient and the dialysis setting [2]. Effective hemodialysis is predicated on the maintenance of circuit patency in the face of numerous factors predisposing to hemostasis and thrombosis (e.g., turbulent blood flow, shear stress, thrombogenic surfaces) [3]. Dialyzer and circuit clotting decreases dialysis quality $[3,4]$.

To reduce the risk of thrombosis and maintain circuit patency, patients on hemodialysis generally receive systemic anticoagulation with heparin. To date, the effectiveness of strategies to avoid systemic anticoagulation with heparin have largely been suboptimal [4-7]. Endexo ${ }^{\mathrm{TM}}$, a novel fluorinated polyurethane surface-modifying macromolecule (SMM), is designed to inhibit the adsorption of protein and platelets [8]. The Endexo additive is currently approved for use in peripherally inserted central catheters $[8,9]$. Thus, it is hypothesized that incorporating Endexo into dialyzer fibers during the 
manufacturing process will improve hemocompatibility and aid in the development of heparin-free hemodialysis. This prospective, open-label clinical study represents the first-in-human use of a dialyzer with Endexo, and aims to assess performance and capture safety information for the dialyzer in adults with ESKD receiving thrice-weekly in-center hemodialysis.

\section{Methods}

\section{Study design and participants}

This prospective, sequential, open-label study was designed to align with US Food and Drug Administration (FDA) guidance on performance testing of new dialyzers [9]. The study was conducted at 3 US hemodialysis centers from August 2018 through April 2019. Eligible patients were aged 22 years or older and had been prescribed in-center thrice-weekly hemodialysis continuously for at least 180 days. Patients were also required to have received hemodialysis treatments with the Optiflux ${ }^{\circledR}$ F160NR dialyzer (manufactured by Fresenius Medical Care North America, Waltham, MA) continuously for at least 30 days prior to entering the study. Other inclusion criteria included: a prescribed hemodialysis treatment time of 3 to 4.5 hours, stable systemic anticoagulation with heparin for at least 2 weeks, hemoglobin concentration of at least $9 \mathrm{~g} / \mathrm{dL}$ and a platelet count of $100,000 / \mathrm{mm}^{3}$ or greater. Patients were excluded from participation if they received hemodialysis with a dialysis acid concentrate containing citric acid.

Patients participated in the study for a maximum of 22 weeks (Fig. 1). Following a screening period of up to 4 weeks, patients underwent 12 hemodialysis treatments with the Optiflux F160NR dialyzer over a span of 4 weeks (Optiflux period). The Endexo period consisted of 38 hemodialysis treatments on the dialyzer with Endexo (approximately 3 times a week over 13 weeks). After the last scheduled hemodialysis treatment in the Endexo period, patients resumed hemodialysis with their previously prescribed dialyzer and attended an in-center follow-up visit within 1 week of the last scheduled study treatment. This study (ClinicalTrials.gov Identifier: NCT03536663) was conducted in accordance with the Principles of the International Council for Harmonisation (ICH) Guideline for Good Clinical Practice (GCP; E6), with approval by an independent Institutional Review Board (Advarra Inc., Columbia, MD, USA and adheres to applicable CONSORT guidelines.

\section{Endpoints}

The selection of endpoints for the present study was based on regulatory guidance from the FDA [9]. The primary endpoint was the in vivo ultrafiltration coefficient (Kuf) of the dialyzer with Endexo during the first study use (visit 13) at 15 minutes ( $\pm 5 \mathrm{~min}$ ) after the recorded start of hemodialysis. This endpoint was derived from machine readings of transmembrane pressure (TMP) and ultrafiltration rate (UFR) and calculated as UFR/TMP.

Secondary endpoints evaluated for both dialyzers included the extent of urea removal (i.e., urea reduction ratio [URR]) and the single-pool Kt/V calculated from pre- and post-hemodialysis blood urea nitrogen 
(BUN) concentrations (spKt/V) for the first study use of each dialyzer. Albumin and $\beta 2$-microglobulin (B2M) concentrations were evaluated before and after hemodialysis treatments for the first study use of each dialyzer. Adverse events (AEs) were coded using Medical Dictionary for Regulatory Activities (MedDRA), Version 20.0, and evaluated as secondary endpoints.

To further characterize the performance of both dialyzers, complement activation (i.e., C3a, C5a, sC5b-9) was assessed before hemodialysis and at 30 minutes $( \pm 10 \mathrm{~min})$ after the recorded start of hemodialysis during the first study use of each dialyzer. At the end of each hemodialysis session, thrombus scoring was performed for each dialyzer using a previously published graded scoring method $[5,10]$. Additional assessments also included URR and spKt/V recorded every 3 to 4 weeks. Finally, data on hemodialysis treatment-related medications (e.g., erythropoiesis-stimulating agents [ESAs], iron, calcitriol), clinical laboratory values, physical examination, and vital signs were recorded.

\section{Analysis}

A sample size of 15 to 24 participants was planned to align with FDA guidance recommending a minimum of 12 patients who each receive at least 36 treatments with the investigational dialyzer [9]. The safety population, used for the evaluation of all safety measurements, was defined as all individuals who had at least one treatment on the dialyzer with Endexo. All other endpoints, including the primary endpoint, were evaluated in the analysis population, defined as all individuals with at least 36 hemodialysis treatments on the dialyzer with Endexo. Continuous endpoints are described with descriptive statistics (i.e., n, mean, standard deviation, median, minimum and maximum), whereas categorical endpoints are described with frequency and percentage. Missing values were not imputed.

To characterize changes in serum albumin and $\beta 2 \mathrm{M}$ associated with each dialyzer, the relative percentage change was calculated. Percentage changes were calculated using corrected posthemodialysis $\beta 2 \mathrm{M}$ concentrations based on work by Bergström and Wehle [11]. In addition to descriptive statistics of complement measures, the relative percentage change (from before dialysis to 30 minutes after the initiation of hemodialysis) was calculated. Thrombus formation is presented as the number of patients and treatments in each period for each grade category (i.e., grade 1 [clear] through 4 [fully clotted]) and as the proportion of treatments with full dialyzer clotting for each patient in each period. AEs and device-related events per treatment were calculated.

\section{Results}

A total of 26 adults provided informed consent and were evaluated for entry into the study; 23 patients were enrolled and received at least one hemodialysis treatment with the Optiflux dialyzer. Per protocol, 4 patients were withdrawn from the study prior to the completion of the Optiflux period as a result of missing a hemodialysis treatment with the Optiflux dialyzer. These 4 patients were aged 62 to 79 years and included 2 men and 2 women. Three of the patients had diabetes as the primary cause of ESKD and one had ESKD secondary to hypertension/large-vessel disease. Of the 19 patients who completed the 
Optiflux period, one patient (a 28-year-old female with ESKD secondary to glomerulonephritis) missed her first treatment on the dialyzer with Endexo (visit 13) and was withdrawn from the trial. The safety population consisted of the 18 patients who received at least one hemodialysis session with the dialyzer with Endexo. As a result of missing multiple treatments with the dialyzer with Endexo secondary to a localized foot infection (not judged to be device-related), one participant was withdrawn from the study per protocol. As such, 17 patients completed a minimum of 36 hemodialysis treatments on the dialyzer with Endexo and made up the analysis population.

\section{Baseline Characteristics And Hemodialysis Treatment Delivered}

The median age of patients in the safety population $(\mathrm{N}=18)$ was 63.5 years (range $27-87$ years). The population was mostly female $(78 \%)$ and identified as white $(67 \%)$. Diabetes was the most common cause of ESKD, accounting for $61 \%$ of cases; hypertension/large-vessel disease, cystic/hereditary/congenital diseases and glomerulonephritis accounted for $22 \%, 11 \%$ and $6 \%$ of cases, respectively.

Initial hemodialysis prescriptions had blood flow rates that ranged from $300 \mathrm{~mL} / \mathrm{min}$ to $500 \mathrm{~mL} / \mathrm{min}$ and dialysate flow rates of $450 \mathrm{~mL} / \mathrm{min}$ to $800 \mathrm{~mL} / \mathrm{min}$. In the Optiflux period, 23 patients received a total of 268 hemodialysis treatments, and 18 participants received 664 hemodialysis treatments in the Endexo period. In the analysis population, delivered hemodialysis treatment parameters were comparable across treatment periods (Table 1 ).

Table 1

Delivered hemodialysis for the analysis population ( $\mathrm{N}=17$ completers)

\begin{tabular}{|lll|}
\hline Measure & Optiflux & Endexo \\
\hline Blood pump flow rate, $\mathrm{mL} / \mathrm{min}$ & $447.5(32.3)$ & $447.7(37.8)$ \\
\hline Dialysate flow rate, $\mathrm{mL} / \mathrm{min}$ & $698.0(60.7)$ & $698.5(62.9)$ \\
\hline Treatment time, min & $205.2(19.1)$ & $207.5(20.5)$ \\
\hline Blood volume processed, L & $82.6(8.1)$ & $82.8(10.7)$ \\
\hline Ultrafiltration volume, $\mathrm{mL}$ & $2156.9(632.6)$ & $2187.1(739.0)$ \\
\hline Data presented as mean (SD) & & \\
\hline
\end{tabular}

\section{Primary And Secondary Endpoints}

In the Endexo period, the mean (SD) Kuf of the dialyzer with Endexo at visit 13 was 15.85 (10.33) $\mathrm{mL} / \mathrm{h} / \mathrm{mmHg}$ (primary endpoint). This in vivo value was derived from a mean (SD) UFR of 652.35 
(232.34) $\mathrm{mL} / \mathrm{h}$ and mean TMP of $46.25(15.44) \mathrm{mmHg}$ observed at 15 minutes ( $\pm 5 \mathrm{~min}$ ) after the recorded start of hemodialysis at visit 13 in the analysis population. At the same session, mean (SD) dialysis adequacy (i.e., spKt/V) was 1.98 (0.56) and URR was 79.59\% (12.15).

Using data across the entirety of each treatment period, the mean (SD) spKt/V for the Optiflux dialyzer and dialyzer with Endexo were $1.94(0.3)$ and 2.09 (0.41), respectively. Treatment with the dialyzers was associated with comparable mean (SD) URRs in the Optiflux (80.81\% [4.33]) and Endexo (81.87\% [5.91]) periods.

Mean (SD) concentrations of $\beta 2 \mathrm{M}$ before hemodialysis were similar at visit 1 (Optiflux) and visit 13 (Endexo): 32.29 (8.37) $\mu \mathrm{g} / \mathrm{mL}$ and $32.9(6.55) \mu \mathrm{g} / \mathrm{mL}$, respectively. Hemodialysis treatment with the dialyzer with Endexo resulted in corrected mean (SD) $\beta 2 \mathrm{M}$ removal rates that were numerically $47 \%$ higher than those observed with the Optiflux dialyzer: $67.73 \%$ (16.32). Mean (SD) pre-dialysis serum albumin levels were comparable for all visits and both dialyzers, ranging from $3.91(0.21) \mathrm{g} / \mathrm{dL}$ (visit 22; Endexo) to $3.97(0.34) \mathrm{g} / \mathrm{dL}$ (visit 1; Optiflux). Albumin levels before dialysis generally remained constant across the Endexo period (mean [SD] of 3.95 [0.21], 3.91 [0.21], 3.94 [0.25] and 3.91 [0.31] g/dL at visits $13,22,34$ and 46, respectively). Treatment with both dialyzers resulted in similar increases in serum albumin levels (Fig. 2).

During the 4-week Optiflux period, 4 out of 23 patients reported a total of $7 \mathrm{AEs}$. This equates to a rate of 2.6 AEs per 100 hemodialysis sessions. No single AE was reported by more than one patient during the Optiflux period, and none of the events were considered device-related. During the Endexo period, 11 patients reported a total of $32 \mathrm{AEs}$, for a rate of $4.8 \mathrm{AEs}$ per 100 hemodialysis sessions. The most commonly reported AEs (those occurring in $\geq 2$ patients) in the Endexo period were headache, reported by 3 patients (16.7\%; 0.5 events per 100 hemodialysis sessions), and lethargy, hypotension, vomiting and extremity pain, each reported by 2 patients (11.1\%; 0.3 events per 100 hemodialysis sessions). None of the AEs experienced in the Endexo period were considered device-related. Three serious AEs were reported during the study (gastrointestinal hemorrhage, localized infection and hypertensive emergency) and all occurred during the Endexo period. None of the serious AEs were considered device- or procedure-related. Throughout the trial, there were no AEs that led to study discontinuation and no deaths.

\section{Additional Endpoints}

The impact of dialysis on hematologic parameters (i.e., hemoglobin, hematocrit and red blood cell count) was evaluated before and after the first study use of the dialyzer with Endexo. There was an absence of clinically significant changes in these parameters. Patients exhibited a mean (SD) hemoglobin increase of $0.49(0.93) \mathrm{g} / \mathrm{dL}$ after the first use of the dialyzer with Endexo. A mean (SD) platelet count decrease of $6.47(13.94) \times 10^{3} / \mu \mathrm{L}$ equating to a $2.7 \%$ (5.70) reduction (from a pre-dialysis mean [SD] of 198.94 [55.85] $\times 10^{3} / \mu \mathrm{L}$ ) was observed after use of the dialyzer with Endexo. 
Protocol-driven modifications for hemodialysis treatment-related medications were excluded, so investigators were permitted to alter medications based on clinical judgment and institutional guidelines. Relative to observations during the Optiflux period, the mean dose of heparin was reduced by $11.2 \%$ during the Endexo period (mean [SD]: 4507 [3349] IU vs 3760 [2471] IU). In a post hoc analysis that excluded 18 hemodialysis sessions from 6 patients with extremely high (i.e., $\geq 10,000$ IU) and extremely low (i.e., 0 IU) recorded heparin doses, the dialyzer with Endexo was associated with a $3.7 \%$ mean reduction in heparin doses. Relative to treatment during the Optiflux period, patients received lower doses of ESA (12.0\% mean reduction) and higher doses of intravenous iron sucrose (13.4\% mean increase) during the Endexo period. In contrast, the mean administered doses of calcitriol, doxercalciferol and saline were comparable across treatment periods (i.e., varied $<10 \%$ ).

Complement activation was assessed at the first visit during each treatment period, with measurements taken before hemodialysis and 30 minutes after the treatment began. There was no evidence of overt complement activation, as $\mathrm{C} 5 \mathrm{a}$ and $\mathrm{C} 3 \mathrm{a}$ levels remained largely unchanged. A slight increase in sC5b-9 was observed for both dialyzers, with mean increases from $260 \mathrm{ng} / \mathrm{mL}$ to $323 \mathrm{ng} / \mathrm{mL}$ during the Optiflux period and $224 \mathrm{ng} / \mathrm{mL}$ to $304 \mathrm{ng} / \mathrm{mL}$ during the Endexo period.

Thrombus scoring was performed at the end of dialyzer use for every treatment session. Scoring was identified as grade 1 (good clear dialyzer, no detectable clotting), 2 (overall light redness, minimal clot formation), 3 (overall moderate redness, clot formation but hemodialysis was still possible) or 4 (total clotting of the dialyzer necessitating a stop in treatment or replacement of the dialyzer). The mean (SD) thrombus scores in the Optiflux and Endexo periods were $1.14(0.4)$ and $1.29(0.52)$, respectively. A single grade 4 thrombus score was recorded during the study, and it occurred during the Optiflux period.

\section{Discussion}

In this prospective, sequential, multicenter, open-label clinical study, the performance of the novel dialyzer with Endexo was comparable to the Optiflux dialyzer, and use of the dialyzer was well tolerated. The mean in vivo Kuf for the dialyzer was calculated as $15.85 \mathrm{~mL} / \mathrm{h} / \mathrm{mmHg}$. Although not evaluated in the present study, in vitro testing has demonstrated that the Kuf of the dialyzer with Endexo is $81 \mathrm{~mL} / \mathrm{h} / \mathrm{mmHg}[12]$.

Discrepancies between the in vitro and in vivo performance of a dialyzer highlight pronounced differences in the testing modalities. Laboratory testing is performed under controlled conditions, often using animal blood with a constant hematocrit and specified total protein level, at UFRs between 600 and $1800 \mathrm{~mL} / \mathrm{h}[9,13]$. In contrast, in vivo testing is subject to the interpatient variability of the cohort. For instance, at visit 13 in the present study, UFRs ranged from $310 \mathrm{~mL} / \mathrm{h}$ to $1020 \mathrm{~mL} / \mathrm{h}$ and TMP ranged from $20 \mathrm{mmHg}$ to $80 \mathrm{mmHg}$. It is also worth noting that Kuf itself generally varies during an HD session [13].

In the present study, the dialyzer with Endexo appeared to be $47 \%$ more efficient at removing $32 \mathrm{M}$ than the Optiflux dialyzer $(67.73 \%)$. Accumulation of $\beta 2 \mathrm{M}$ in patients on long-term hemodialysis can result in 
deposition of amyloid (i.e., dialysis-related amyloidosis) in musculoskeletal and cardiac tissue [14, 15]. Higher clearance rates of $\beta 2 \mathrm{M}$ have been linked with reduced (or delayed) rates of dialysis-related amyloidosis [16]. Among patients receiving hemodialysis, higher $\beta 2 \mathrm{M}$ levels are predictive of increased mortality, and this association is independent of duration of ESKD, diabetes, malnutrition and chronic inflammation [17-19]. Moreover, evidence suggests that greater removal of $\beta 2 \mathrm{M}$ may serve as a biomarker of reduced cardiovascular mortality among individuals receiving maintenance hemodialysis [17]. Although direct comparisons cannot be made, the $\beta 2 \mathrm{M}$ removal rate of the dialyzer with Endexo is greater than that traditionally reported for high-flux dialysis and comparable to that reported for mediumcutoff dialyzers [20].

Both the Optiflux and the dialyzer with Endexo examined in this study resulted in similar increases in serum albumin concentrations after hemodialysis. Notably, pre-dialysis albumin concentrations remained relatively constant across the 13-week Endexo period. This stability can be contrasted with recent data from a study of a medium-cutoff dialyzer demonstrating significant reductions (relative to a high-flux dialyzer) in serum albumin levels over a 3-month period [21]. Among patients undergoing hemodialysis, reduced levels of serum albumin are a strong predictor of mortality, but the clinical significance of dialysis-associated reductions in albumin concentrations, in the absence of reductions in nutritional status, remains unclear [21, 22].

Although the dialyzer with Endexo is being developed for future potential heparin-free therapy, the present trial was not designed to evaluate the effects of the dialyzer on heparin use. Investigators were not asked to follow any study-specific anticoagulant protocols, and other components of the dialysis system did not incorporate the Endexo polymer. The reduction in heparin doses observed during the Endexo period was markedly attenuated when a small number of sessions with outlier (recorded) heparin doses were excluded from the analysis. The data from the present study suggest that the dialyzer with Endexo had no clinically relevant impact on hematologic parameters. The observed reductions in ESA doses during the Endexo period likely contributed to the observed increases in intravenous iron use and may reflect temporal changes in anemia management at the centers. Finally, there is a recognized association between reduced ESA needs and lower levels of inflammation as assessed by serum C-reactive protein levels [23]. Future studies should continue to explore the effects of the dialyzer with Endexo on anemia therapies and should examine inflammatory biomarkers.

\section{Conclusions}

This study represents the first clinical study of a novel dialyzer containing the SMM Endexo blended into the membrane during manufacturing and was designed to align with FDA guidance on performance testing of new dialyzers [9]. Although the number of patients receiving treatment during the Endexo period was modest $(\mathrm{N}=18)$, more than 660 hemodialysis treatments were delivered during the 13-week period. As the trial was not designed to directly compare the investigational dialyzer with the Optiflux dialyzer, formal statistical analysis comparing the treatments was not conducted. 
In summary, the performance of the novel dialyzer with Endexo was generally comparable to that of the Optiflux dialyzer while exhibiting a higher $\beta 2 \mathrm{M}$ removal rate. The dialyzer with Endexo was well tolerated and demonstrated no evidence of overt complement activation. Additional studies are needed to explore whether this novel dialyzer can be incorporated into a heparin-free hemodialysis system to provide an alternative to systemic anticoagulation.

\section{Abbreviations}

AE: Adverse event; $\beta 2 \mathrm{M}$ : $\beta 2$-microglobulin; BUN: Blood urea nitrogen; ESKD: End-stage kidney disease; ESA: Erythropoiesis-stimulating agent; GCP: Guideline for Good Clinical Practice; ICH: International Council for Harmonisation; MedDRA: Medical Dictionary for Regulatory Activities; SMM: Surfacemodifying macromolecule; TMP: Transmembrane pressure; UFR: Ultrafiltration rate; URR: Urea reduction ratio; FDA: US Food and Drug Administration

\section{Declarations}

Ethics approval and consent to participate: The clinical study protocol, written informed consent form, and all amendments that required approval were reviewed by an independent Institutional Review Board (Advarra Inc., Columbia, MD, USA). Prior to admission into the study, each subject received a comprehensive explanation of the proposed treatment and provided written informed consent to participate.

Consent for publication: Not applicable

Availability of data and materials: All data requests should be submitted to the corresponding author for consideration.

Competing interests: AAZ, MT, CHH, SA, CM and RJK are or were employees of Fresenius Medical Care Renal Therapies group during the study.

Funding: The study was sponsored by Fresenius Medical Care Renal Therapies Group, Waltham, MA, USA.

Authors' contributions: JMM, DS and LAW were principal investigators and contributed to the study conceptualization, patient enrollment and follow-up and data analysis. AAZ contributed to the study conceptualization, investigation and methodology. MT contributed to the study conceptualization, investigation, methodology, resources, supervision, data analysis and writing. $\mathrm{CHH}$ contributed to the study conceptualization, investigation, methodology, data management and biostatistics supervision and writing. SA contributed to the study conceptualization, methodology and safety analysis. CM contributed to the study conceptualization, investigation, methodology, resources, supervision, data analysis, writing and editing. RJK contributed to the study conceptualization, investigation, resources and supervision. All authors read and approved the final manuscript. 
Acknowledgements: Medical writing and editing support was provided by Adam Perahia, MD, of NorthStar Strategic Consulting, LLC, via funding by Fresenius Medical Care Renal Therapies Group.

\section{References}

1. Liyanage T, Ninomiya T, Jha V, et al. Worldwide access to treatment for end-stage kidney disease: a systematic review. Lancet. 2015;385:1975-82.

2. Haroon S, Davenport A. Choosing a dialyzer: what clinicians need to know. Hemodial Int. 2018;22:65-74.

3. Fischer KG. Essentials of anticoagulation in hemodialysis. Hemodial Int. 2007;11:178-89.

4. Islam MS, Hassan ZA, Chalmin F, et al. Vitamin E-coated and heparin-coated dialyzer membranes for heparin-free hemodialysis: a multicenter, randomized, crossover trial. Am J Kidney Dis. 2016;68:75262.

5. Laville M, Dorval M, Fort Ros J, et al. Results of the HepZero study comparing heparin-grafted membrane and standard care show that heparin-grafted dialyzer is safe and easy to use for heparinfree dialysis. Kidney Int. 2014;86:1260-7.

6. Meijers BK, Poesen R, Evenepoel P. Heparin-coated dialyzer membranes: is non-inferiority good enough? Kidney Int. 2014;86:1084-6.

7. Shen JI, Winkelmayer WC. Use and safety of unfractionated heparin for anticoagulation during maintenance hemodialysis. Am J Kidney Dis. 2012;60:473-86.

8. Lopez-Donaire ML, Santerre JP. Surface modifying oligomers used to functionalize polymeric surfaces: consideration of blood contact applications. J Appl Polym Sci. 2014;131:40328.

9. US Department of Health and Human Services, Food and Drug Administration, Center for Devices and Radiological Health. Guidance for Industry and CDRH Reviewers: Guidance for the content of premarket notifications for conventional and high permeability hemodialyzers. 07 August 1998. Accessed 18 Mar 2020.

10. Dorsch O, Krieter DH, Lemke HD, et al. A multi-center, prospective, open-label, 8-week study of certoparin for anticoagulation during maintenance hemodialysis-the membrane study. BMC Nephrol. 2012;13:50.

11. Bergström J, Wehle B. No change in corrected beta 2-microglobulin concentration after cuprophane haemodialysis. Lancet. 1987;1:628-9.

12. Data on file. Fresenius Medical Care Renal Therapies Group LLC. Waltham, MA; 2019.

13. Ficheux A, Kerr PG, Brunet $P$, Argilés À. The ultrafiltration coefficient of a dialyser (KUF) is not a fixed value, and it follows a parabolic function: the new concept of KUF max. Nephrol Dial Transplant. 2011;26:636-40.

14. Morris AD, Smith RN, Stone JR. The pathology and changing epidemiology of dialysis-related cardiac beta-2 microglobulin amyloidosis. Cardiovasc Pathol. 2019;42:30-5.

15. Fukuda K, Yamamoto H. Dialysis-related amyloidosis. Semin Musculoskelet Radiol. 2001;5:113-9. 
16. Kaneko S, Yamagata K. Hemodialysis-related amyloidosis: is it still relevant? Semin Dial. 2018;31:612-8.

17. Argyropoulos $\mathrm{CP}$, Chen SS, $\mathrm{Ng} \mathrm{YH}$, et al. Rediscovering beta-2 microglobulin as a biomarker across the spectrum of kidney diseases. Front Med (Lausanne). 2017;4:73.

18. Cheung AK, Rocco MV, Yan G, et al. Serum $\beta$-2 microglobulin levels predict mortality in dialysis patients: results of the HEMO Study. J Am Soc Nephrol. 2006;17:546-55.

19. Okuno S, Ishimura E, Kohno K, et al. Serum beta2-microglobulin level is a significant predictor of mortality in maintenance haemodialysis patients. Nephrol Dial Transplant. 2009;24:571-7.

20. Masakane I, Sakurai K. Current approaches to middle molecule removal: room for innovation. Nephrol Dial Transplant. 2018;33(suppl 3):iii12-21.

21. Belmouaz $M$, Bauwens $M$, Hauet $T$, et al. Comparison of the removal of uraemic toxins with medium cut-off and high-flux dialysers: a randomized clinical trial. Nephrol Dial Transplant. 2020;35:328-35.

22. Ma L, Zhao S. Risk factors for mortality in patients undergoing hemodialysis: a systematic review and meta-analysis. Int J Cardiol. 2017;238:151-8.

23. Chawla LS, Krishnan M. Causes and consequences of inflammation on anemia management in hemodialysis patients. Hemodial Int. 2009;13:222-34.

\section{Figures}

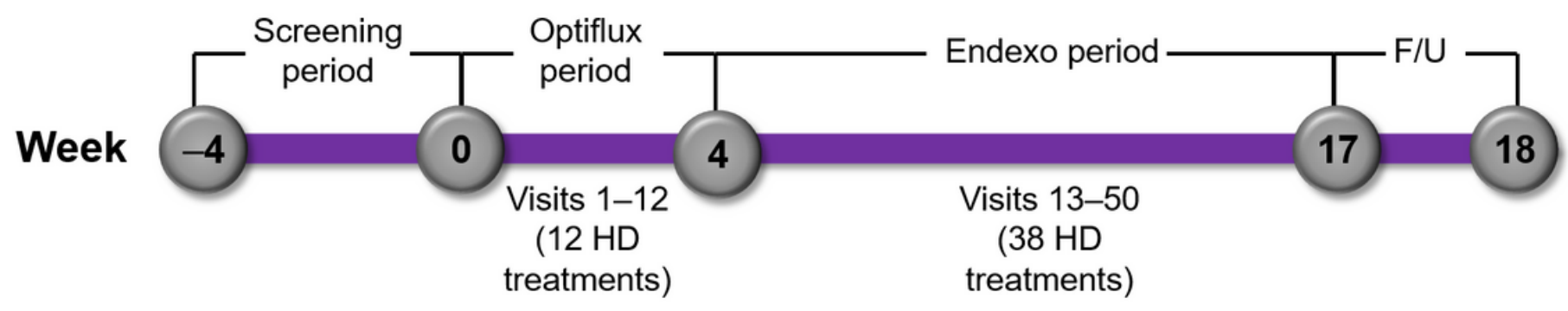

\section{Figure 1}

Study design F/U follow-up, HD hemodialysis 


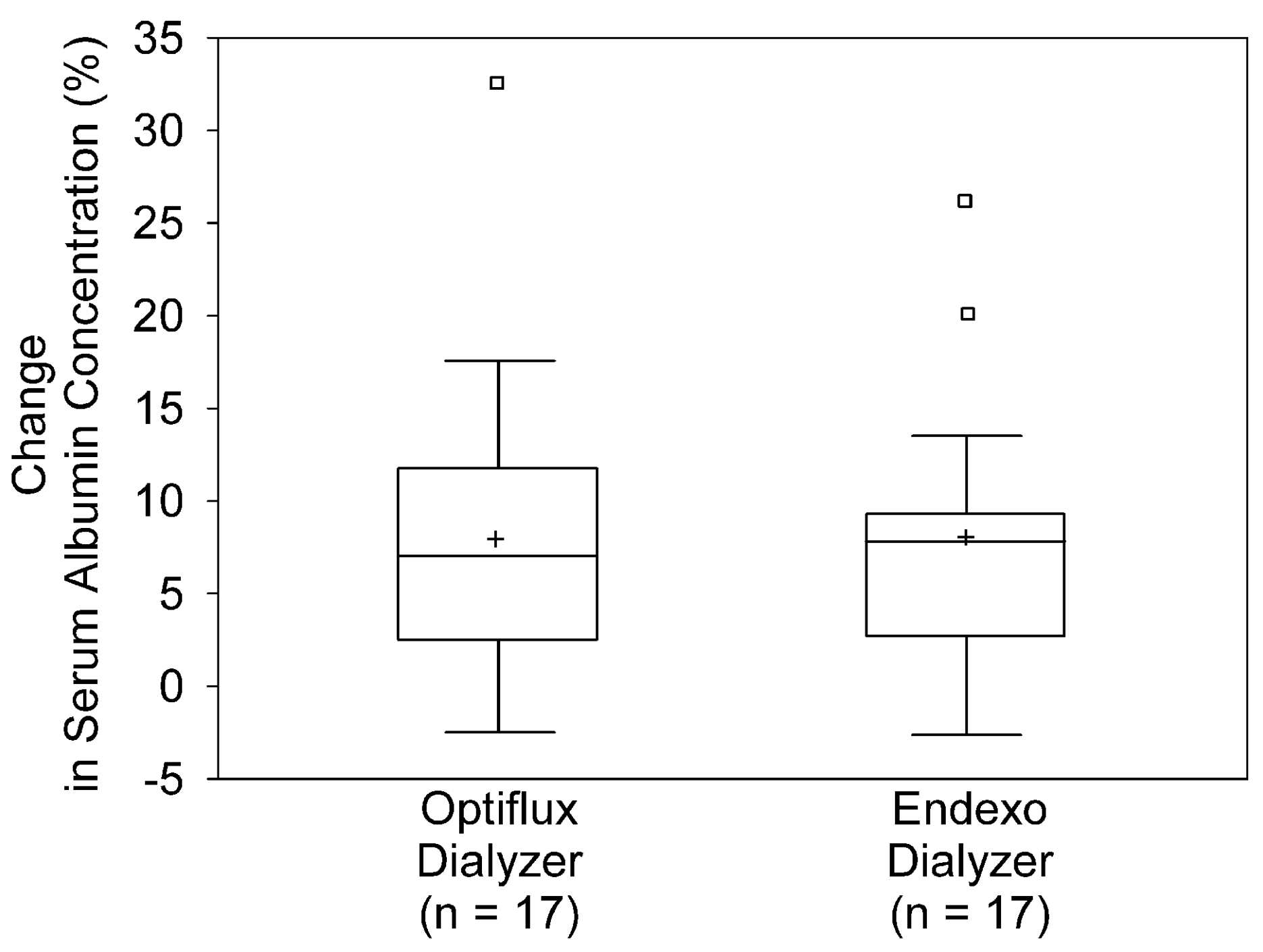

Figure 2

Box plot of percentage changes in serum albumin concentration by dialyzer (pre-HD to post-HD) in the analysis population Percent change $=($ Post HD - Pre-HD $) /$ Pre-HD $* 100$ HD hemodialysis, IQR interquartile range Box represents interquartile range and median (horizontal line); whiskers represent adjacent values (i.e., highest value within Q3 + [1.5 x IQR] and lowest value within Q1 + [1.5 x IQR]); + represents mean value; $\square$ represents outlier value

\section{Supplementary Files}

This is a list of supplementary files associated with this preprint. Click to download.

- CONSORT2010ChecklistforBMCNephrology042620.doc 